Electronic Supporting Information for:

\title{
The effect of the anomeric configuration on the micellization of hexadecylmaltoside surfactants
}

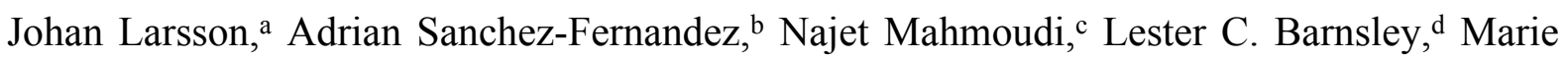
Wahlgren, ${ }^{\text {b,e }}$ Tommy Nylander, ${ }^{\text {,,f }}$ Stefan Ulvenlund ${ }^{\text {be }}$

aPhysical Chemistry, Department Chemistry, Lund University, Box 124, 22100 Lund, Sweden.

${ }^{b}$ Food Technology, Nutrition and Engineering, Lund University, Box 124, 22100 Lund, Sweden.

'ISIS Spallation Source, STFC, Rutherford Appleton Laboratory, Didcot, UK.

dü̈lich Centre for Neutron Science (JCNS) at Heinz Maier-Leibnitz Zentrum (MLZ), Forschungszentrum Jülich GmbH, Lichtenbergstr. 1, 85748 Garching, Germany.

eEnza Biotech AB, Getingevägen 60, 22241 Lund, Sweden.

fNanoLund, Lund University, Lund, Sweden 



\section{HPLC characterization of the surfactants}

The surfactants used in this work were characterized using HPLC. The chromatograms resulting from the measurements are presented in Figure S1 and the values calculated of purity are included in the Experimental section of the main text.

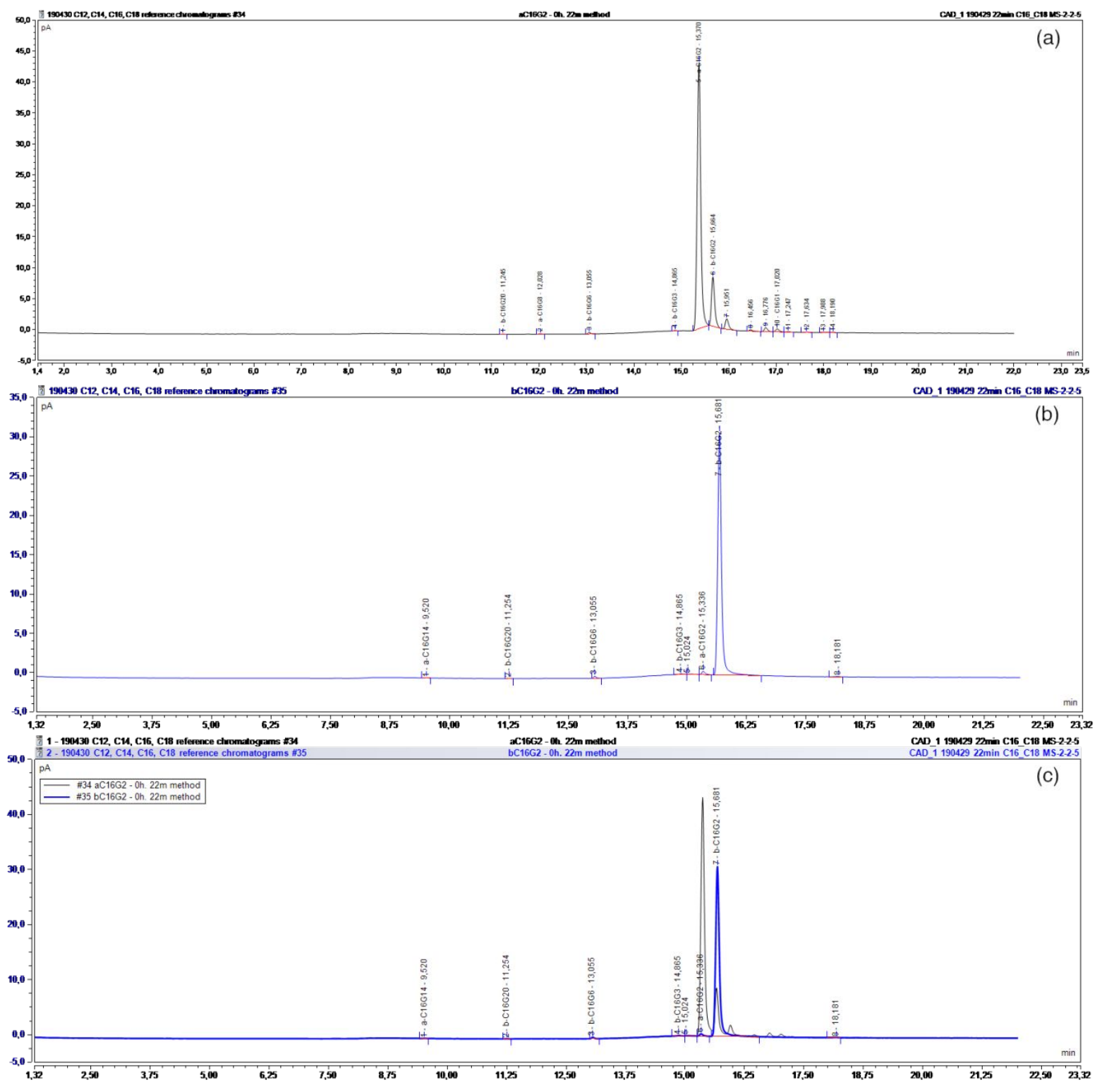

Figure S1 HPLC chromatograms of (a) $\alpha-\mathrm{C}_{16} \mathrm{G}_{2}$ and (b) $\beta-\mathrm{C}_{16} \mathrm{G}_{2}$. For comparison, (c) shows the overlap of (black line) $\alpha-\mathrm{C}_{16} \mathrm{G}_{2}$ and (blue line) $\beta-\mathrm{C}_{16} \mathrm{G}_{2}$ chromatograms.

\section{Dynamic light scattering}

Results from dynamic light scattering (DLS) measurements of $\alpha-C_{16} G_{2}$ and $\beta-C_{16} G_{2}$ at different concentrations and temperatures are presented in Figure $\mathrm{S} 2$ and Figure $\mathrm{S} 3$, respectively. The hydrodynamic radius was obtained by fitting the experimental autocorrelation function using the Cumulant method. 


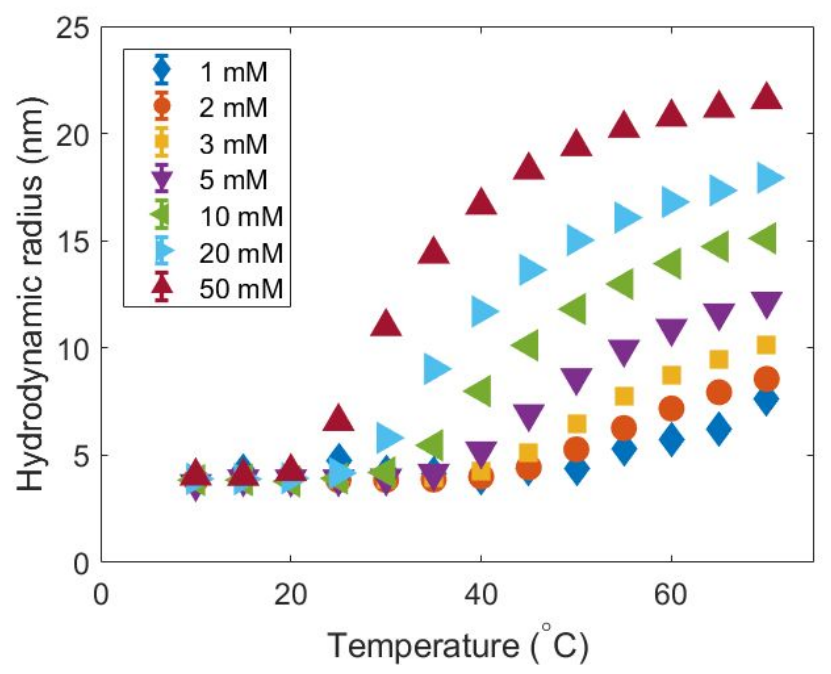

Figure S2 Hydrodynamic radius of $\alpha-\mathrm{C}_{16} \mathrm{G}_{2}$ micelles versus temperature at different concentrations.

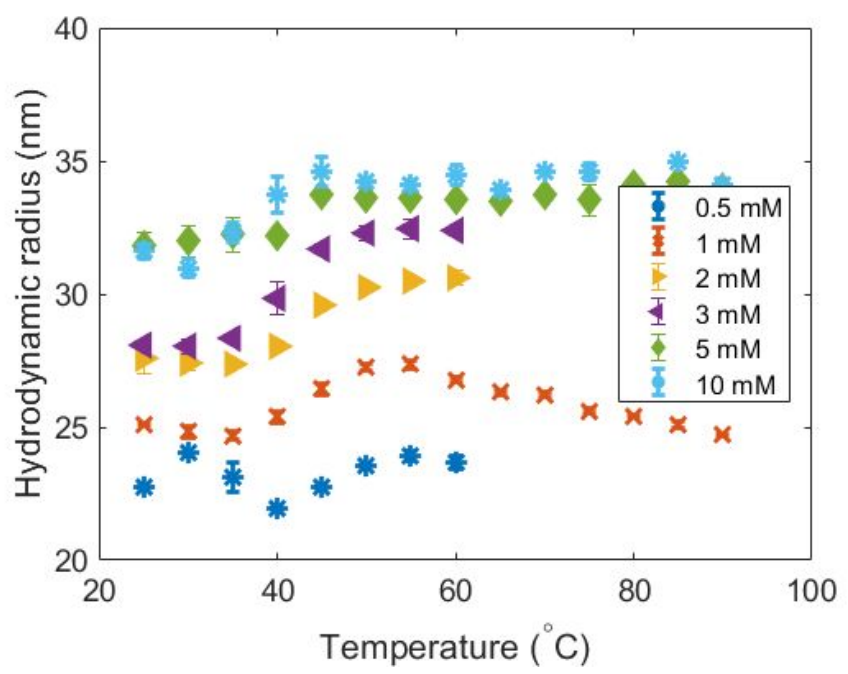

Figure S3 Hydrodynamic radius of $\beta-\mathrm{C}_{16} \mathrm{G}_{2}$ micelles versus temperature at different concentrations.

As shown by these results, the effect of temperature and concentration appear to differ between the two surfactants. A detailed description of these results is found in the main text.

\section{Solvent isotope effect on micelle structure}

As presented in the main manuscript, isotope substitution tends to affect the structure of the surfactant aggregates. The small-angle neutron scattering measurements presented here were performed in $\mathrm{D}_{2} \mathrm{O}$, and since previous investigations have revealed a quite substantial effect of $\mathrm{D}_{2} \mathrm{O}$ on alkylglycoside micelle size, ${ }^{1}$ we have evaluated the effect of isotope substitution by means of DLS. The results from these experiments are presented in Figure S4, where the hydrodynamic radius of protonated $\beta-\mathrm{C}_{16} \mathrm{G}_{2}$ micelles in $\mathrm{D}_{2} \mathrm{O}$ is compared to that in $\mathrm{H}_{2} \mathrm{O}$ at different temperatures. 


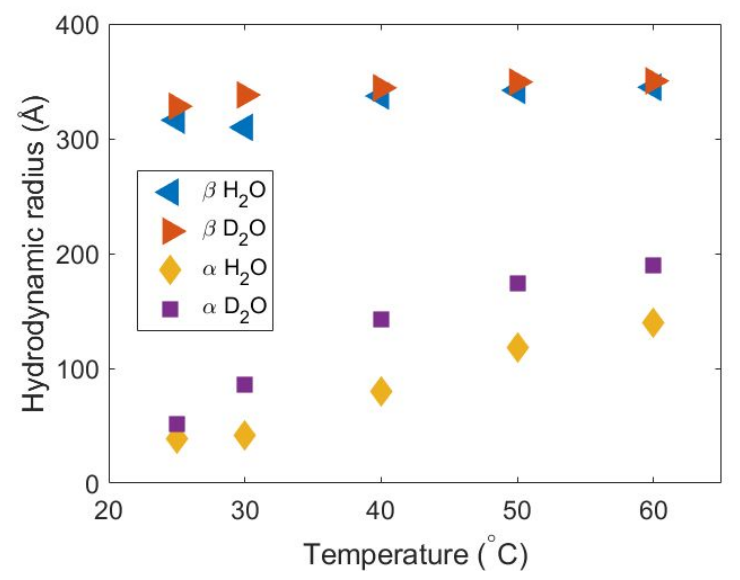

Figure S4 Hydrodynamic radius of $\alpha-\mathrm{C}_{16} \mathrm{G}_{2}$ and $\beta-\mathrm{C}_{16} \mathrm{G}_{2}$ micelles versus temperature for 10 $\mathrm{mM}$ samples in $\mathrm{H}_{2} \mathrm{O}$ and $\mathrm{D}_{2} \mathrm{O}$ at different temperatures.

In aqueous solution, the hydrodynamic radius of the micelles was found to be slightly larger in $\mathrm{D}_{2} \mathrm{O}$ than in $\mathrm{H}_{2} \mathrm{O}$, which demonstrates that the substitution of protonated solvent for deuterated solvent has an impact on the overall size of the surfactant aggregates. In the case of $\alpha-\mathrm{C}_{16} \mathrm{G}_{2}$, the differences between the two solvents were found to be significant, in some cases $50 \%$ larger when the surfactant self-assembles in $\mathrm{D}_{2} \mathrm{O}$. Furthermore, and despite the differences in size, the morphology transition observed for the alpha anomer is maintained when changing the solvent, with micelles growing at higher temperatures. For $\beta-C_{16} G_{2}$, the micelles are still larger in $\mathrm{D}_{2} \mathrm{O}$, although difference in size is less pronounced than in the case of the alpha configuration. The hydrodynamic radius of the micelles as measured by DLS was found to be around $5 \%$ larger in the case of the deuterated solvent.

In order to quantify differences occurring at molecular level as a result of the isotopic substitution, small-angle X-ray scattering (SAXS) measurements at high momentum transfer $\left(>0.01 \AA^{-1}\right)$ were performed using an in-house SAXSLAB's Ganesha 300 XL. The SAXS curves and models from such measurements are presented in Figure S5, and the results from those fits are presented in Table S1.

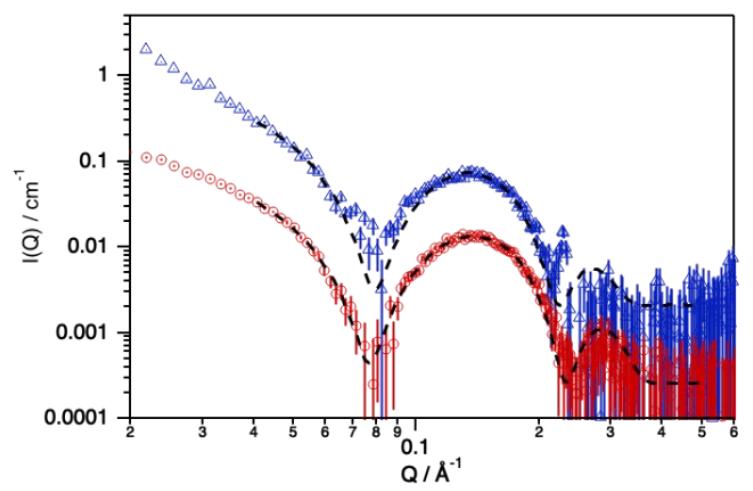

Figure S5 SAXS data and fits of $10 \mathrm{mM}$ of $\beta-\mathrm{C}_{16} \mathrm{G}_{2}$ in (red circles) $\mathrm{H}_{2} \mathrm{O}$ and (blue triangles) $\mathrm{D}_{2} \mathrm{O}$ at $50{ }^{\circ} \mathrm{C}$. The black dashed lines represent the best fits using a core-shell cylinder model. Data and fits have been offset for clarity.

Table S1 Results from the fits of SAXS data of $10 \mathrm{mM}$ of $\beta-\mathrm{C}_{16} \mathrm{G}_{2}$ in $\mathrm{H}_{2} \mathrm{O}$ and $\mathrm{D}_{2} \mathrm{O}$ at $50{ }^{\circ} \mathrm{C}$, presented in Figure S4. The elongation of the micelle was fixed at $4300 \AA$ and significant variations in this value were not found to affect the results presented here. 


\begin{tabular}{|c|c|c|c|}
\hline System & $\mathrm{r}_{\text {core }} / \AA$ & $\mathrm{t}_{\text {shell }} / \AA$ & $\mathrm{SLD}_{\text {shell }} / 10^{-6} \AA^{-2}$ \\
\hline $\mathrm{H}_{2} \mathrm{O}$ & $16.2 \pm 0.3$ & $14.2 \pm 0.2$ & $10.4 \pm 0.2$ \\
\hline $\mathrm{D}_{2} \mathrm{O}$ & $16.1 \pm 0.3$ & $15.9 \pm 0.1$ & $10.6 \pm 0.1$ \\
\hline
\end{tabular}

The SAXS analysis of the surfactant solution in two different isotopic solvents shows differences in the cross-section of the micelle. The micelle core appears to remain unaffected by the substitution of the solvent. Differences can be however found in the characteristics of the headgroup region, where the surfactant solvated in $\mathrm{H}_{2} \mathrm{O}$ shows a thickness around $12 \%$ smaller than that for the surfactant in $\mathrm{D}_{2} \mathrm{O}$. The SLD of the shell is also slightly different but such a difference remains within the experimental error.

This demonstrates that the exchange of $\mathrm{H}_{2} \mathrm{O}$ for $\mathrm{D}_{2} \mathrm{O}$ will have an effect on the scattering data that we use to determine the morphology of the micelles. Although the main trends are preserved with varying the isotopic composition of the solvent, the interpretation of the results shall be carefully approached. The discussion regarding this matter can be found in the main text.

\section{Small-angle scattering}

The small-angle scattering data presented in the main text were fitted using a core-shell density distribution for an ellipsoid, cylinder and flexible cylinder, depending on the elongation of the micelle. A detailed list of the parameters from these fits is presented in Table S2, S3, S4 and S5. These parameters were subsequently used to calculate the results presented in the main text.

Table S2 Fitting parameters derived from the analysis of small-angle scattering data of $\alpha$ $\mathrm{C}_{16} \mathrm{G}_{2}$ at different concentrations of surfactant and $50{ }^{\circ} \mathrm{C}$.

\begin{tabular}{|c|c|c|c|c|c|c|}
\hline$\left[\alpha-\mathrm{C}_{16} \mathrm{G}_{2}\right] / \mathrm{mM}$ & $\mathrm{r}_{\text {core }} / \AA$ & $\mathrm{t}_{\text {shell }} / \AA$ & $\mathrm{L}_{\text {core }} / \AA$ & $\mathrm{lp} / \AA$ & $\varphi_{\text {fit }} / 10^{-2}$ & $\mathrm{SLD}_{\text {shell }} / 10^{-6} \AA^{-2}$ \\
\hline 1 & $14.7 \pm 0.3$ & $20.7 \pm 0.2$ & $86 \pm 3$ & - & $0.14 \pm 0.03$ & $9.90 \pm 0.05$ \\
\hline 2 & $14.7 \pm 0.3$ & $18.3 \pm 0.2$ & $150 \pm 10$ & - & $0.24 \pm 0.02$ & $10.00 \pm 0.05$ \\
\hline 3 & $14.7 \pm 0.3$ & $17.4 \pm 0.2$ & $220 \pm 10$ & - & $0.35 \pm 0.02$ & $10.05 \pm 0.02$ \\
\hline 5 & $14.7 \pm 0.3$ & $14.6 \pm 0.2$ & $270 \pm 10$ & - & $0.41 \pm 0.02$ & $10.19 \pm 0.01$ \\
\hline 10 & $14.7 \pm 0.3$ & $14.4 \pm 0.2$ & $520 \pm 10$ & - & $0.78 \pm 0.01$ & $10.24 \pm 0.01$ \\
\hline 20 & $14.7 \pm 0.3$ & $14.1 \pm 0.1$ & $650 \pm 10$ & - & $1.46 \pm 0.01$ & $10.28 \pm 0.01$ \\
\hline
\end{tabular}

Table S3 Fitting parameters derived from the analysis of small-angle scattering data of $\alpha$ $\mathrm{C}_{16} \mathrm{G}_{2}$ at $10 \mathrm{mM}$ surfactant concentration and different temperatures.

\begin{tabular}{|c|c|c|c|c|c|c|}
\hline $\mathrm{T} /{ }^{\circ} \mathrm{C}$ & $\mathrm{r}_{\text {core }} / \AA$ & $\mathrm{t}_{\text {shell }} / \AA$ & $\mathrm{L}_{\text {core }} / \AA$ & $\mathrm{lp} / \AA$ & $\varphi_{\text {fit }} / 10^{-2}$ & $\mathrm{SLD}_{\text {shell }} / 10^{-6} \AA^{-2}$ \\
\hline 25 & $14.7 \pm 0.3$ & $21.8 \pm 0.4$ & $81 \pm 3$ & - & $1.01 \pm 0.01$ & $9.90 \pm 0.06$ \\
\hline 40 & $14.7 \pm 0.3$ & $17.7 \pm 0.2$ & $230 \pm 10$ & - & $0.91 \pm 0.01$ & $10.05 \pm 0.07$ \\
\hline 50 & $14.7 \pm 0.3$ & $14.4 \pm 0.2$ & $570 \pm 10$ & - & $0.78 \pm 0.01$ & $10.24 \pm 0.01$ \\
\hline
\end{tabular}

Table S4 Fitting parameters derived from the analysis of small-angle scattering data of $\beta$ $\mathrm{C}_{16} \mathrm{G}_{2}$ at different concentrations of surfactant and $50{ }^{\circ} \mathrm{C}$.

\begin{tabular}{|c|c|c|c|c|c|c|}
\hline$\left[\beta-\mathrm{C}_{16} \mathrm{G}_{2}\right] / \mathrm{mM}$ & $\mathrm{r}_{\text {core }} / \AA$ & $\mathrm{t}_{\text {shell }} / \AA$ & $\mathrm{L} / \AA$ & $\mathrm{lp} / \AA$ & $\varphi_{\text {fit }} / 10-2$ & $\mathrm{SLD}_{\text {shell }} / 10^{-6} \AA^{-2}$ \\
\hline 1 & $16.1 \pm 0.1$ & $15.4 \pm 0.2$ & $>2000$ & - & $0.07 \pm 0.02$ & $10.34 \pm 0.06$ \\
\hline 2 & $16.1 \pm 0.1$ & $15.1 \pm 0.2$ & $>2000$ & - & $0.13 \pm 0.09$ & $10.37 \pm 0.04$ \\
\hline 3 & $16.1 \pm 0.1$ & $15.3 \pm 0.1$ & $>2000$ & - & $0.20 \pm 0.05$ & $10.35 \pm 0.04$ \\
\hline 5 & $16.1 \pm 0.1$ & $15.2 \pm 0.1$ & $>2000$ & - & $0.32 \pm 0.04$ & $10.36 \pm 0.02$ \\
\hline 10 & $16.1 \pm 0.1$ & $15.1 \pm 0.1$ & $>2000$ & - & $0.63 \pm 0.03$ & $10.36 \pm 0.01$ \\
\hline 18 & $16.1 \pm 0.1$ & $15.1 \pm 0.1$ & $6700 \pm 200$ & $310 \pm 10$ & $1.11 \pm 0.02$ & $10.36 \pm 0.01$ \\
\hline
\end{tabular}


Table S5 Fitting parameters derived from the analysis of small-angle scattering data of $\beta$ $\mathrm{C}_{16} \mathrm{G}_{2}$ at $18 \mathrm{mM}$ surfactant concentration and different temperatures.

\begin{tabular}{|c|c|c|c|c|c|c|}
\hline $\mathrm{T} /{ }^{\circ} \mathrm{C}$ & $\mathrm{r}_{\text {core }} / \AA$ & $\mathrm{t}_{\text {shell }} / \AA$ & $\mathrm{L}_{\text {core }} / \AA$ & $2 \mathrm{lp} / \AA$ & $\varphi_{\text {fit }} / 10^{-2}$ & $\mathrm{SLD}_{\text {shell }} / 10^{-6} \AA^{-2}$ \\
\hline 25 & $16.2 \pm 0.1$ & $15.3 \pm 0.3$ & $>2000$ & - & $1.11 \pm 0.02$ & $10.39 \pm 0.06$ \\
\hline 40 & $16.2 \pm 0.1$ & $15.2 \pm 0.1$ & $>2000$ & - & $1.11 \pm 0.02$ & $10.37 \pm 0.07$ \\
\hline 50 & $16.1 \pm 0.1$ & $15.1 \pm 0.1$ & $6700 \pm 200$ & $310 \pm 10$ & $1.10 \pm 0.02$ & $10.36 \pm 0.01$ \\
\hline
\end{tabular}

\section{Reference}

1. Ericsson, C. A.; Soderman, O.; Garamus, V. M.; Bergstrom, M.; Ulvenlund, S., Effects of temperature, salt, and deuterium oxide on the self-aggregation of alkylglycosides in dilute solution. 2. n-Tetradecyl-beta-D-maltoside. Langmuir 2005, 21 (4), 1507-15. 